\title{
Arzneimittelverschreibung wird ein Stück komplizierter
}

\begin{abstract}
_ Der Bundestag hat am 9. März 2017 das Gesetz zur Stärkung der Arzneimittelversorgung in der GKV (AMVSG) beschlossen. Die abschließende Beratung im Bundesrat hatte zu Redaktionsschluss noch nicht stattgefunden. Die wesentlichen Teile des Gesetzes sollen noch im April 2017 in Kraft treten. Betroffen ist v. a. die Pharmaindustrie. So
\end{abstract}

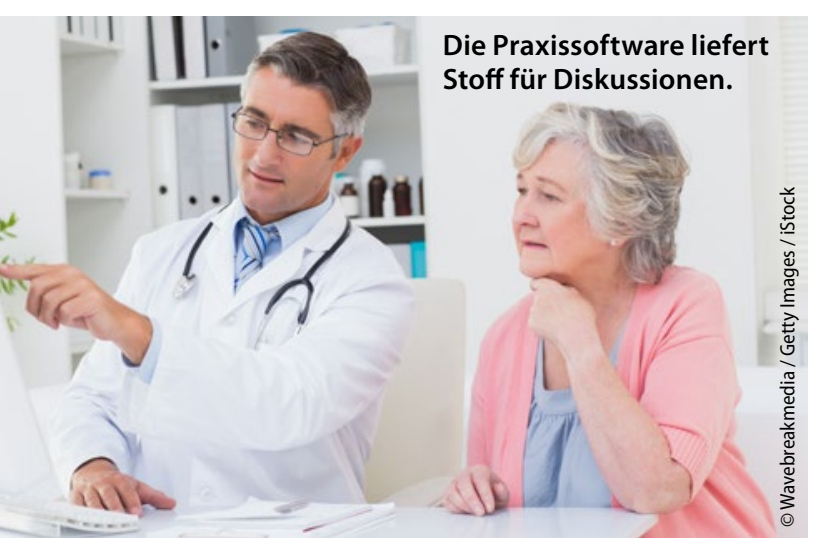

erhalten private Krankenversicherer, Beihilfe- und Heilfürsorgeträger gegenüber den Pharmaherstellern einen gesetzlichen Anspruch, dass die Differenz zwischen dem Erstattungsbetrag und dem tatsächlichen Abgabepreis ausgeglichen wird. Das könnte die Beitragssteigerungen in der PKV dämpfen. Am Rande sind aber auch die Praxen unmittelbar von dem Gesetz betroffen und hier wird es interessant.

\section{MMW-KOMMENTAR \\ Wegen der zunehmenden Antibioti- karesistenzen soll die Erstattung von diagnostischen Verfahren, die einen zielgenaueren Einsatz der MIttel er- möglichen, im EBM geregelt werden. Gedacht ist dabei an Schnellanalyse- verfahren, wie sie schon heute $z$. B. zur Bestimmung des CRP-Werts ver- wendet werden. Die Kassen werden es im Bewertungsausschuss künftig}

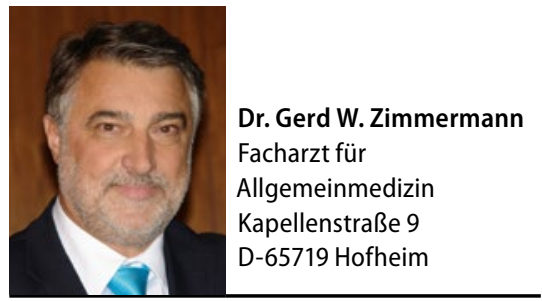

schwerer haben, Dumping-Preise für solche Laboranalysen durchzusetzen.

Weniger hilfreich dürfte hingegen eine gesetzliche Neuerung sein, die die Praxissoftware betrifft. Hier sollen in Zukunft die Ergebnisse der Nutzenbewertung von Arzneimitteln besser sichtbar werden, damit die Ärzte sie bei den Therapieentscheidungen berücksichtigen können. Ensprechende gesetzliche Vorgaben gab es schon bisher, doch ist das Bundesgesundheitsministerium unzufrieden mit der Art und Weise, in der die KBV diese umzusetzen versucht. Nun könnte das Ministerium bald konkret vorgeben, wie die Infos genau abgebildet werden müssen. Dies dürfte zu einer weiteren Teuerungswelle bei der Praxis-EDV und einem neuerlichen Bürokratieschub in den Praxen führen. Wir dürfen uns auch auf vermehrte Diskussionen mit betroffenen Patienten einrichten, die nicht wahrhaben wollen, dass die Medikamente, die sie haben wollen, in der Nutzenbewertung "durchgefallen" sind.

\section{Neues zur häuslichen Palliativpflege}

— Mit zwei praxisrelevanten Beschlüssen hat der Gemeinsame Bundesausschuss (G-BA) am 16. März 2017 die Richtlinie zur häuslichen Krankenpflege (HKP) geändert. Verbessert werden soll ärztliche Behandlung sterbender Menschen in ihrer Häuslichkeit, bei denen keine spezialisierte ambulante Palliativversorgung (SAPV) erforderlich ist. Dafür wird die neue Nr. 24a in die HKPRichtlinie eingeführt.

Die Leistung ermöglicht es Pflegediensten, die Symptome der Palliativpatienten selbst zu kontrollieren - wenn auch in enger Abstimmung mit dem ver- ordnenden Arzt. Konkret geht es um Schmerzen, Übelkeit, Erbrechen, pulmonale oder kardiale Symptome, Obstipation sowie die Kontrolle und Behandlung exulzerierender Wunden. Auch Kriseninterventionen etwa bei Krampfanfällen, Blutungen oder akuten Angstzuständen wird den Pflegern durch die Neuerung ermöglicht.

Palliativpflege kann jeder Vertragsarzt schwerstkranken und sterbenden Patienten jeden Alters verordnen. Dafür muss eine nicht heilbare Erkrankung vorliegen, die nach fachlicher Einschätzung des behandelnden Arztes so weit fortgeschritten sein muss, dass die Lebenserwartung auf Tage oder wenige Wochen limitiert ist. Primär sollen nun die Beschwerden gelindert und die Lebensqualität verbessert werden.

Die Dauer der Erst- und Folgeverordnungen beträgt jeweils bis zu 14 Tage. Die Häufigkeit richtet sich nach dem individuellen Bedarf - die Anzahl der täglichen Pflegeeinsätze ist also nicht beschränkt.

\section{MMW-KOMMENTAR}

Diese Neuerungen im Rahmen der allgemeinen ambulanten Palliativversorgung (AAPV) 